\title{
BRONJ In A Patient with Rheumatoid Arthritis: An Unnamed Association
}

\author{
Yusuf Ziya Şener ${ }^{1 *}$, Burak Yasin Aktaş ${ }^{1}$, Levent Kılıç̧2, Ahmet Çağkan İnkaya ${ }^{3}$ and Umut Kalyoncu ${ }^{2}$ \\ ${ }^{1}$ Department of Internal Medicine, Hacettepe University, Turkey \\ ${ }^{2}$ Department of Rheumatology, Hacettepe University, Turkey
}

${ }^{3}$ Department of Infectious Diseases, Hacettepe University, Turkey

Received: July 10, 2017; Published: July 14, 2017

*Corresponding author: Yusuf Ziya Şener, Faculty of Medicine, Department of Internal Medicine, Hacettepe University, Turkey, Tel: +9050585988 53;Email: yzsener@yahoo.com.tr

Abstract

Bisphosphonates (BPs) are important drugs in treatment and prevention of all types of osteoporosis. BRONJ is a well known complication of BP treatment since 2002 when it was firstly described. Although most of the reported cases are patients with malignancies. Rheumatoid arthritis is a well known risk factor for BRONJ. In this report, we present a patient with BRONJ who was in our follow up due to rheumatoid arthritis.

\section{Introduction}

Bisphosphonates (BPs) are drugs that used to treat and prevent from cancer and drug related osteoporosis. BPs are analogous of inorganic pyrophosphate and separated into two sub groups either containing nitrogen group. BPs bind to bones and reduce the activity of osteoclasts. Bisphosphonate related osteo necrosis of jaw (BRONJ) is a well defined complication of treatment with BPs. BRONJ is defined as existence of exposed necrotic bone in the oral cavity lasting more than 8 weeks without any radiation exposure and the presence of BP use history [1]. Here in we present a female patient with rheumatoid arthritis who was complicated with BRONJ while her follow up.

\section{Case Report}

A 67 years old female patient with rheumatoid arthritis (RA), hypertension and osteoporosis admitted to our Rheumatology outpatient clinics with the complaint of jaw pain 3 years ago. She had been diagnosed RA 20 years before due to arthritis of symmetrical meta carpophalangeal joints and bilateral knees with morning stiffness, elevated inflammatory marks (erythrocyte sedimentation rate and C-reactive protein) and positive rheumatoid factor level. Her disease was under control with rituximab and DMARD combination (Leflunomide plus hydroxyl chloroquine). Her other medications were prednisolone $5 \mathrm{mg} /$ day and alendronate $70 \mathrm{mg} /$ week. It is learnt that she had been using alendronate since 9 years.

Her physical examination was normal except slight tenderness at her jaw region. Complete blood count revealed mild normochromic normocyticanemia with thrombocytosis consistent with chronic inflammatory state. Her kidney and liver function tests were normal. Erythrocyte sedimentation rate was $34 \mathrm{~mm} /$ $\mathrm{hr}$ and C-ractive protein (CRP) level was $3.2 \mathrm{mg} / \mathrm{dL}(0-0.8 \mathrm{mg} / \mathrm{dL})$. It is learnt that a tooth extraction was performed 1 year ago and prosthetic tooth was implanted just after extraction. 6 months after tooth implantation, prosthetic tooth revision was performed due to infection. Although she had used multiple antibiotics to heal her gingivitis and tooth infection her complaints persisted during this period. She was referred to a dentist to be examined in case of the complication of implanted tooth and infection.

Draining pus was detected from left premolar tooth region was detected in her dental examination and necrotic bone lining under implanted tooth was debrided and the specimen was sent to pathological evaluation. Osteonecrosis with ostemomyelitis was detected and actinomyces colonies were growth from anaerobic tissue culture. The patient was diagnosed as BRONJ due to the history of bisphosphonate use and referred to Infectious Disease Department. Amoxicillin clavulanate was prescribed and patient was followed monthly dental examination with ESR and CRP levels. After 6 months therapy her jaw tenderness were relieved, ESR and CRP levels returned to normal range. She is still in our follow up for RA.

\section{Discussion}

BRONJ is defined as presence of exposed bone in the oral cavity lasting more than 8 weeks with the history of BP use. BRONJ is 
staged into 4 sub groups that stage 0 describes no clinical evident bone necrosis with the existence of symptoms such as jaw pain, tenderness etc. and stage 3 describes exposed bone with pain and swelling in conjunction with radio graphically demonstrated osteolysis that requires surgical debridement with antibiotic use while stage 1 and stage 2 defines the intermediate clinical conditions between stage 0 and stage 3 . The incidence of BRONJ in patients with malignancy house BPs by intravenous route; is between $1-18 \%$ changing with malignancy type [1]. It is known that the amount of treatment time with BPs also has an impact on BRONJ incidence. Kühl et al. [2] reported that BRONJ incidence is $0-11.5 \%$ at the end of 1 year treatment while it is $0-27.5 \%$ at the end of 4 years with treatment [2].

Smoking, drinking, obesity, invasive dental treatments, treatment duration, drug dose, presence of underlying malignancy, rheumatoid arthritis, diabetes, end stage renal disease and co-administration of corticosteroids, anti-angiogenic agents (thalidomide, bevacizuamb etc.) and tyrosine kinase inhibitors are well known risk factors for BRONJ. Nitrogen containing BPs have more risk for BRONJ development than non-nitrogen containing BPs. All patients who are planned to prescribed BPs should. AllpatientswhoareplannedtoprescribedBPsshould be referred to dental examination. Although there are controversies about drug holiday before invasive dental procedures; it will be reasonable to stop treatment 2 months before the intervention in patients who are under treatment with BPs for more than 4 years by the lights of literature. Treatment should contain antibacterial mouth wash in all stages and surgical debridement in advanced stages [3].

Increased frequency of periodontal diseases, chronic inflammatory state, corticosteroid and metorexate use are the main causes of higher incidence of BRONJ in patients with RA. DiFede et al. reported that female gender, age older than 60 years and increased duration of BPs use are related with BRONJ development in RA patients [4].

\section{Conclusion}

To conclude, BRONJ is a rare but serious complication of treatment with BPs and it should be suspected in RA patients with jaw pain and tenderness who have a history of BP use.

\section{References}

1.Paulo S, Abrantes AM, Laranjo M, Carvalho L, Serra A, et al. (2014) Bisphosphonate-related osteonecrosis of the jaw: specificities. Oncol Rev 8(2): 254 .

2.Kühl S, Walter C, Acham S (2012) Bisphosphonate-related osteonecrosis of the jaws - a review. Oral Oncol 48(10): 938-947.

3.Yoneda T, Hagino H, Sugimoto T (2017) Antiresorptive agent related osteonecrosis of the jaw: Position Paper 2017 of the Japanese Allied Committee on Osteonecrosis of the Jaw. J Bone MinerMetab 35(1): 6-19.

4.DiFede O, Bedogni A, Giancola F (2016) BRONJ in patients with rheumatoid arthritis: a multicenter case series. Oral Dis 22(6): 543-548.

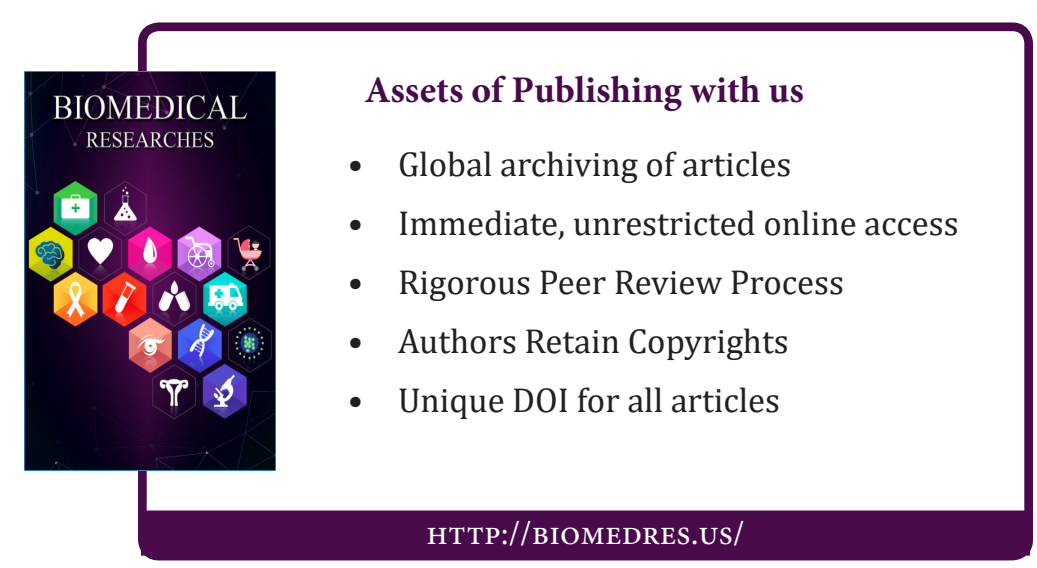

